\title{
The Norwegian Mother and Child Cohort Study (MoBa) - MoBa recruitment and logistics
}

\author{
Patricia Schreuder and Elin Alsaker \\ Norwegian Institute of Public Health, Bergen, Norway \\ Correspondence: elin.alsaker@fhi.no
}

This is an open access article distributed under the Creative Commons Attribution Licence, which permits unrestricted use, distribution, and reproduction in any medium, provided the original work is properly cited.

After some preliminary challenges, the Norwegian Mother and Child Cohort Study (MoBa) started recruitment in one county in June 1999. By 2004 the study was national and included 50 of the 52 hospitals in Norway. The goal of recruiting 100000 pregnancies was reached in May 2008, 9 years after the start. However, nine hospitals continued recruitment until the end of December 2008. More than 114000 children are included in MoBa. The first MoBa child was born in October 1999 and the last in July 2009.

\section{IT-SYSTEMS}

At present $\mathrm{MoBa}$ has two main IT-systems in use: MoBaStudy and MorBarnData. Previously, there were two separate administrative tracking systems, one for recruitment and management of questionnaires and one for management of biological samples. Today, these two systems have been integrated and form the administrative system MoBaStudy. This system keeps track of name, address, email address, mobile phone number and national personal identification number for each $\mathrm{MoBa}$ participant. When questionnaires, consent forms etc. are sent out or returned, this is registered in the system. In the same way, administrative information for MoBa sub-studies is also registered. MoBaStudy is both a logistics system, and a LIMS system (Laboratory Information Management system) for handling biological material.

The second main IT-system, MorBarnData, handles questionnaire data, analysis results from biological samples and other health information. Each questionnaire is marked with a unique barcode consisting of questionnaire-type code, child number and a unique study-id for each pregnancy. The child number is relevant in the case of twins and triplets.

The two IT-systems are logically integrated through a numeric identifier but physically separate. Both systems are generic and may be adapted for use in similar type of studies.

\section{RECRUITMENT}

Participant recruitment relied upon close collaboration with maternity units at hospitals throughout Norway. Establishing this collaboration with so many hospitals, however, took several years. In the end, 50 of the 52 hospitals in Norway took part. A few gynecologists in private practice also contributed for a short period. There were no exclusion criteria, but since the ques- tionnaires were only in Norwegian, non-Norwegian speakers are probably under-represented.

All women attending the routine ultrasound examinations at $17 / 18$ weeks at hospitals taking part in recruitment were invited to participate in $\mathrm{MoBa}(98 \%$ of all pregnant women attend ultrasound scans at a hospital). The long recruitment period - almost 10 years, resulted in many women being invited, and consequently participating more than once. The MoBa study unit is therefore pregnancy and not the individual. Each pregnancy was allocated a unique study-id number. In addition, each individual (i.e. mother, father and child) was allocated a unique person-id. The study-id allows linkage between questionnaires related to a specific pregnancy. The linkage between study-id and personid makes it possible to identify siblings.

On the basis of weekly lists sent from the hospitals, the pregnant women were registered in the MoBa tracking database (MoBaStudy). These lists, which were either electronic or postal, included the individual's 11-digit personal identification number, name, address and date for routine ultrasound screening. Approximately three weeks prior to her ultrasound appointment, the woman was sent an invitation package, containing information about the study, a consent form and, in the beginning, the first two questionnaires. Fathers were also invited to participate in the study from 2000. The father's invitation was from then on included in the mother's invitation package. It was then up to the mother to give him the invitation. All those invited were asked to complete and return the informed consent forms and the questionnaires as soon as possible and preferably before attending the ultrasound examination. Midwives at the ultrasound units were able to answer questions and be assured that the consent forms had been returned to MoBa. It was important that consent forms were signed before blood samples were taken. For different reasons a small number of women were not included in the lists sent to us and therefore were not invited prior to their ultrasound appointment. Others had simply not made up their minds by the time of the ultrasound appointment. In these cases, the midwife could give further information and recruit to MoBa. Women, who had not already been invited but signed the consent form at the ultrasound appointment, received an invitation package by mail.

Hospitals informed MoBa about early miscarriages. In such cases, where the invitation had not already been sent to the woman, she was not invited. 
Table 1. Participation in MoBa.

\begin{tabular}{lccc}
\hline & Total recruited & $\begin{array}{c}\text { Participants } \\
\text { per Oct 13 }\end{array}$ & $\begin{array}{c}\text { Active participants } \\
\text { per Oct } 13^{\text {th }} \text { 2014 }\end{array}$ \\
\hline Participating pregnancies - mothers & 112908 & 112768 & 101842 \\
Participating pregnancies - fathers & 87436 & 87332 & 78954 \\
Participating mothers & 95369 & 95249 & 86419 \\
Participating fathers & 75618 & 75526 & 68519 \\
Participating children & 114622 & 114485 & 103574 \\
Sets of twins & 1950 & 1946 & 1715 \\
Sets of triplets & 21 & 21 & 17 \\
\hline
\end{tabular}

A child was registered in MoBaStudy through three alternative sources: 1) cord blood sample, received and registered; 2) birth record in Medical Birth Registry of Norway; or 3) registration in the National Population Registry.

It should be noted that recruitment and follow-up in $\mathrm{MoBa}$ were parallel functions. Thus the logistics during the first 10 years of the study were complicated and resource demanding.

\section{STUDY STATUS}

A status code is given in MoBaStudy to each pregnancy and the persons related to that pregnancy. Status may change over time. When a consent form was returned, the pregnancy status was changed from "Invited" to "Participant" in MoBaStudy. Status codes regulate whether or not participants receive questionnaires or other correspondence from the study. Pregnancy status "Miscarriage" or "Stillbirth" ensured no further contact. A participant may withdraw from the study at any time without giving a reason. If the participant does not specifically request deletion of all information and destruction of biological samples, his or her status is set to "Withdrawn" and data and biological samples from this person may still be included in research. However, if the participant requests that all data and biological samples be deleted/destroyed then information on that person will be erased from MoBaStudy and all data relating to that person will be deleted and samples destroyed.

Despite efforts to ensure that consent forms were signed either before or at the ultrasound appointment, there were still more than 5000 pregnancies in 2010 where blood samples had been donated and/or questionnaires returned without a written consent. However, the Regional Committees for Medical and Health Research Ethics and the Norwegian Data Protection Authority agreed that donating a blood sample or returning a questionnaire could be considered as consent in itself. This meant that the collected material could be used for research purposes. The persons concerned were informed about the decision and asked to contact $\mathrm{MoBa}$ if they wanted their data to be deleted and their samples destroyed. They were also given the opportunity to sign the MoBa's consent form and participate in the study. If no response was received then the person was included as a "passive" participant. Passive parti- cipants receive MoBa's annual newsletter, but are not sent new questionnaires.

$\mathrm{MoBa}$ is regularly linked to the National Population Registry. Through this linkage MoBaStudy is updated with new address information and death of participants. If mail is returned with an unknown address, the status in MoBaStudy is updated to "Moved". If the person later is registered with a new address in the National Population Registry, the address and status is updated in our system. In recent years, we have asked participants for their e-mail addresses and telephone numbers.

\section{Participation}

The total participation rate for $\mathrm{MoBa}$ is $41 \%$. Invitations were sent to women for a total of 277702 pregnancies during the recruitment period. Invitation to the father were included in 259837 of these invitation packages. More than 95000 women, 75000 men and 114000 children are included in MoBa (Table 1).

Due to the long recruitment period, 49500 women were sent invitations for two pregnancies and 6000 for three or more. The participation rate was considerably higher at the start of the study than towards the end. It was also higher for first-time invited than for the total cohort.

Several initiatives have been carried out to increase the participation rate. Information about the study and the contents of the invitation package have been changed several times during the course of the study. In the beginning, the first questionnaire (Q1) and the nutrition questionnaire $(\mathrm{Q} 2)$ were included in the invitation package. Due to criticism from participants about the length of questionnaires and how long it took to complete them, as well as reports from midwives that quite a number of women were reluctant or refused to participate for the same reason, it was decided not to include Q2 in the package. From 2004, Q2 was sent out in gestational week 22 , about 7 weeks after the invitation package. In $2005,50 \%$ of the invited women received the annual newsletter one week prior to their scheduled ultrasound appointments, hoping to create more interest in the study. The women were randomly selected. However, neither splitting the questionnaires into two shipments, nor sending a newsletter, had any measurable effect on the participation rate. 


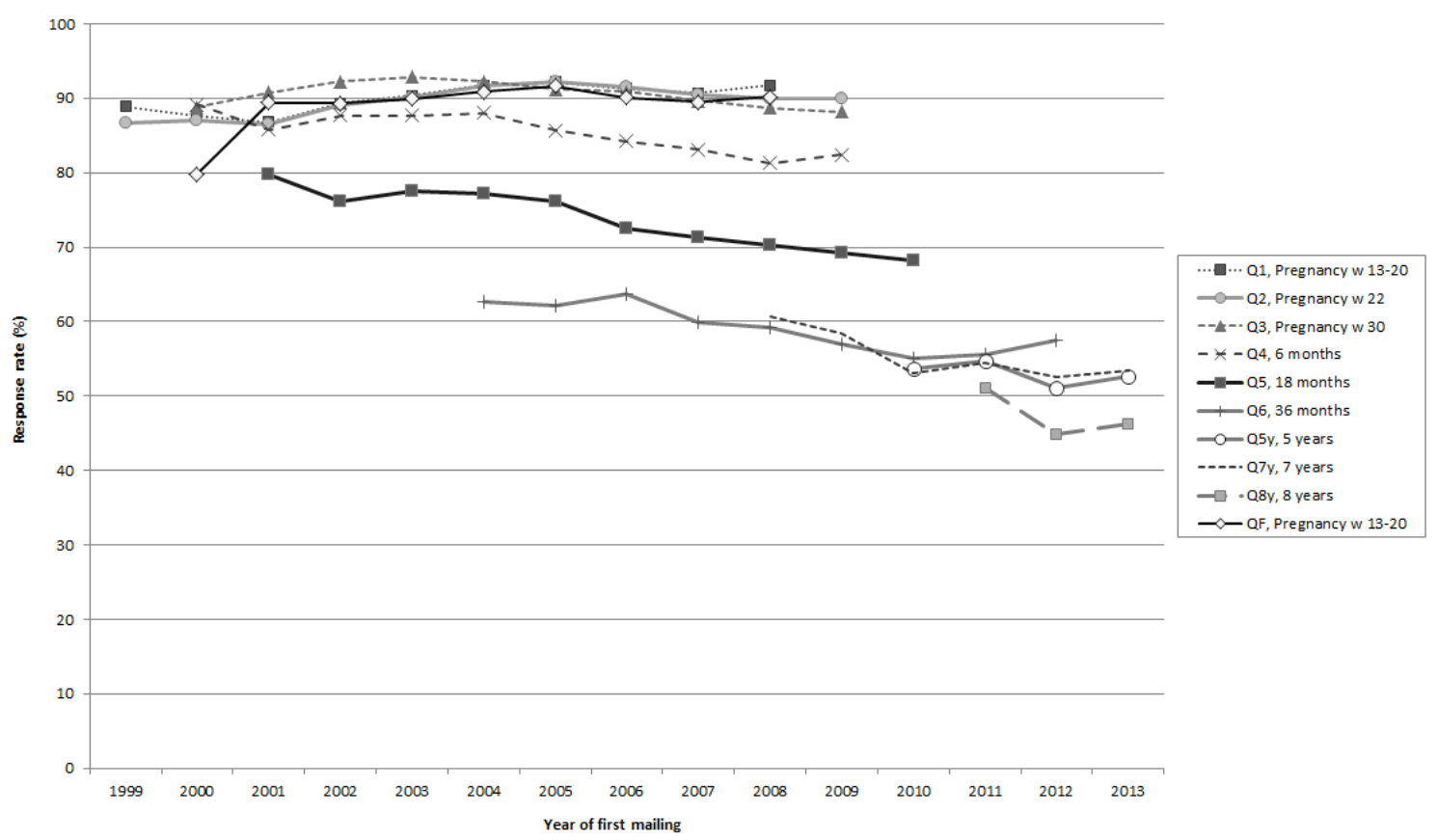

Figure 1. Yearly response rates for MoBa questionnaires.

\section{FOLLOW-UP}

The MoBa-questionnaires are age-specific and so far there are 10 postal questionnaires: three were sent to the mothers during pregnancy at 17 weeks, 22 weeks and 30 weeks and one to the father. Questionnaires were then sent to the mother when the baby was 6 and 18 months and again when the child was 3 years, 5 years, 7 years and 8 years. Mothers of twins or triplets receive one questionnaire for each child. Electronic versions have been tried out for some questionnaires over a period of time, where the mother was given the option of completing the questionnaire either on paper or online. Only $17 \%$ chose to complete the nutrition questionnaire online and $13 \%$ the 3 year questionnaire. Admittedly, this was early in the study when financial and technical resources were limited. In 2010, a professional web-designer developed an online version of the 7 year questionnaire. The result was disappointing - a slight decrease in the total response rate for the 7 year questionnaire. Thus, the online alternative was stopped. Given the choice, it seems that most mothers in $\mathrm{MoBa}$ prefer to fill out a paper version of the questionnaires.

There were several revised versions of the first questionnaires. This has complicated the creation of data files and researchers' interpretation of the data. Aware of the consequences, we are now reluctant to make changes in questionnaires once they are in use.

At present, postal questionnaires are being sent to mothers of 7 and 8 year olds. Almost $70 \%$ receive reminders, resulting in extra high printing and postage costs. One questionnaire for the mother and one for the child is planned at age 13 , in addition to a dietary questionnaire for the child at age 14. A follow-up questionnaire for the father is under development.
In 2008 we started collecting milk teeth when the child is approximately 7 years old. So far MoBa has received 20,800 teeth.

Several sub-projects, such as the Autism Birth Cohort Study $(\mathrm{ABC})$, the ADHD Study and the Language and Learning Study (SOL) have collected additional data through clinical examinations.

The response rate for the questionnaires during pregnancy, including the father's questionnaire, was consistently high and although the rate has dropped for each subsequent questionnaire, the rate for each age specific questionnaire has been rather stable throughout the study $(\mathrm{Q} 1=91 \%$; Q7y $=54 \%$; Q8y $=47 \%$, Figure 1).

\section{INCENTIVES}

Attrition is a challenge in all longitudinal studies. To motivate the participants to fill in questionnaires several incentives have been introduced. A height chart was enclosed with the 18 month or the three year questionnaire but no increase in response was found. Other incentives and improvements, such as a childfriendly brochure included with the 7 year questionnaire, Christmas card together with reflector bands sent to all participants, newsletters (both postal and email), extra text-reminders, shorter questionnaires, improved website (http://www.fhi.no/morogbarn) have all been attempted without significant effect.

\section{Data Management}

Returned questionnaires are scanned using Readsoft Eyes and Hands Forms. This automated program reads, interprets and validates data using predefined rules. The program produces semicolon separated data files 


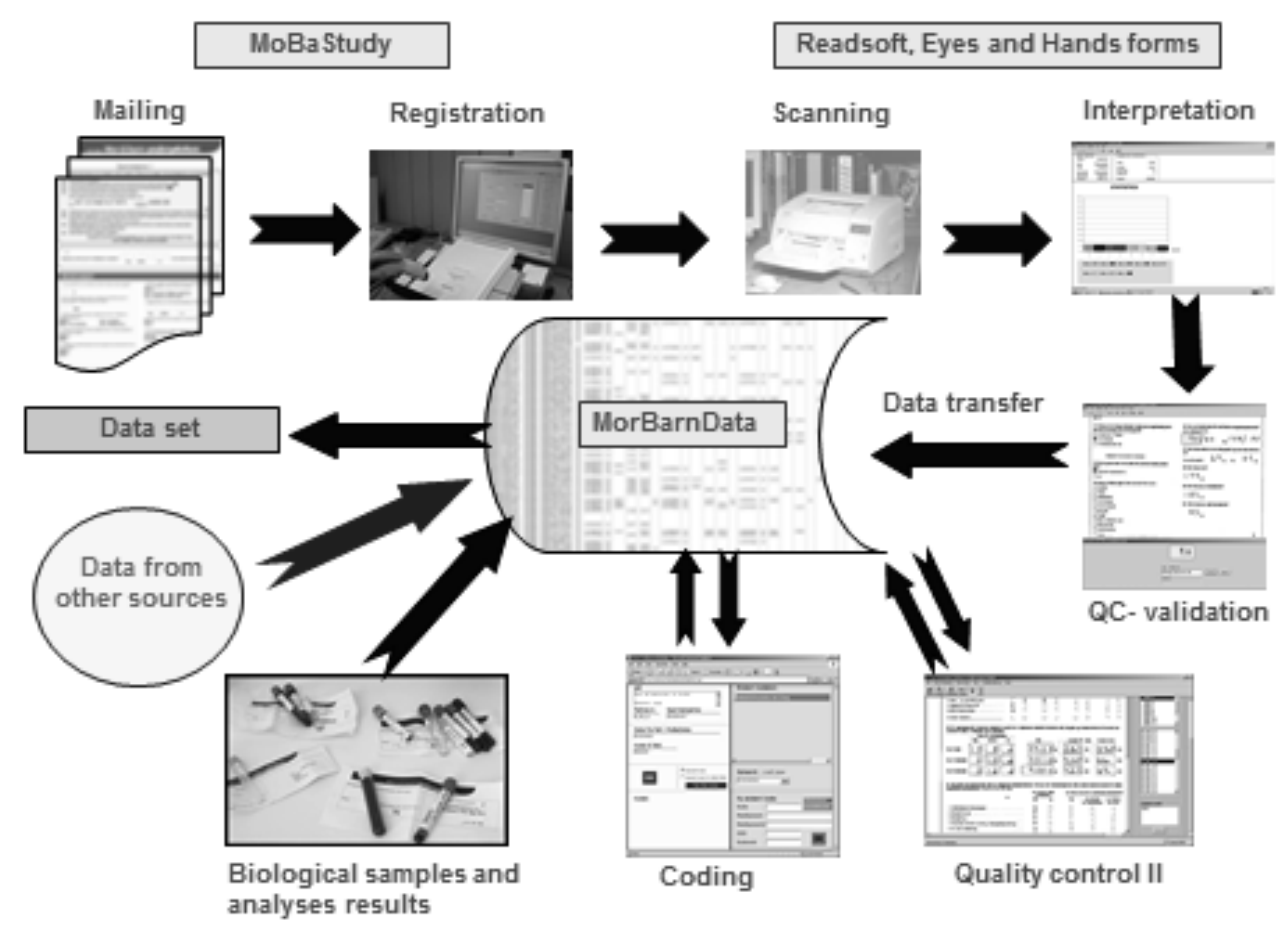

Figure 2. Data management.

which are imported into MorBarnData. Questionnaires are also stored as optical images.

The data is further checked in MorBarnData for possible invalid values such as values outside a given range, as well as inconsistencies between fields. If there are discrepancies between what is written in the questionnaire and data registered in the database, corrections are made. Otherwise data are left unchanged. Medicines and occupations are coded using standardized coding manuals. Data from MoBa is linked to the Medical Birth Registry of Norway (MBRN). Dependent on permission from the Regional Committees for Medical and Health Research Ethics (REK), MoBa data may also be linked to other registries such as the Cancer Registry of Norway, the Norwegian Patient Registry (NPR), the Norwegian Prescription Database, the Cause of Death Registry of Norway and the Norwegian Immunization Registry (SYSVAK).
A complete data set containing all questionnaire data is released once a year.

\section{THE FUTURE}

Today, new technological methods within data collection are available. Despite our previous experience with web-based questionnaires we are now exploring solutions for implementing a web-based alternative. The first children in the MoBa cohort are now 15 years old. When they are 18, they will be asked for their consent to participate in MoBa. Future questionnaires will then be addressed to them. Smart telephones and tablets will, most likely, be their chosen instrument for filling out questionnaires. Social media, such as Facebook and Twitter will be useful for keeping in touch with the youngsters. To encourage participation, MoBa must strive to keep up with time and development.

\section{APPENDIX, IT-SYSTEMS}

The MoBaStudy is both a logistics system for the administration of participants, including dates for dispatch and registration of questionnaires, and a LIMS system (Laboratory Information Management System) for handling biological material. It is also used to keep track of invitations and participation in MoBa-sub studies and clinical examination. MoBaStudy has dual language support.

Some features of MoBaStudy:

- Dynamic merge letters defined by users

- Questionnaire dispatches based on user defined rules for (sub-) population and timing of each dispatch

- Biological sample management

- Biological sample retrieval

- Communication with laboratory robots

- Reports - both standard and user defined

- All user interface labels, menus and messages are defined in the database 
- User rights (visibility, update access) to all interface components are defined in the database

- Change/audit log for most data fields (can easily redefine which ones are audited)

- Code 128 Barcode labels for questionnaires, sample tubes, racks, shelves and freezers

- PDA version for Sample retrievals - PDALab

- Communication with RTS A4 Sample-Store ${ }^{\mathrm{TM}}$

MorBarnData is a system for handling questionnaire data, analyses results from biological samples and other health information. The database contains data for answers from all the returned questionnaires. It also contains all question and answer alternative text from the questionnaires both in Norwegian and English (Metadata). MorBarnData is designed and developed using an Oracle 9.2.0.5 database on an HP Itanium server with HP Unix 11i.

MorBarnData contains:

- Full Metadata set in English and Norwegian.

- Questionnaire data.

- Location coordinates for all fields in the questionnaires.

- Data load logs and error logs.

- Quality control data and rules.

- Tracking of changes made to data.

- Medicine and occupation codes.

- User rights.

- Reports

- Independent number-series per project (used for data delivery)

The front end for this database is a client application also designed and developed using Visual Studio .Net C\#.

This application is used to:

- Dynamically define new questionnaires based on Readsoft Eyes \& Hands Forms description files

- Dynamically add new tables and fields in MorBarnData to store the questionnaire data.

- Keep track of questionnaire type and link revised versions of each type

- Load scanned questionnaires.

- View the questionnaire images and compare to scanned data.

- Quality control system / tracking of all changes.

- Graphically define and modify quality control rules.

- Graphical data extraction (data shopping list), automatically creates SAS and SPSS files with metadata (variables and values labels) based on the shopping list (selected questions). 